\title{
Facilitating Adaptive Tutorial Dialogues in EER-Tutor
}

\author{
Amali Weerasinghe, Antonija Mitrovic \\ Intelligent Computer Tutoring Group, University of Canterbury, New Zealand \\ amali.weerasinghe@pg.canterbury.ac.nz,tanja.mitrovic@canterbury.ac.nz
}

EER-Tutor is a constraint-based intelligent tutoring system that teaches conceptual database design. Students are provided a problem solving environment to design a data model for a real world scenario. We enhanced EER-Tutor with adaptive tutorial dialogues to facilitate discussion of mistakes in a student solution. The dialogues discuss errors in the current problem context as well as the relevant domain concepts. The dialogues are customised based on the student model.

Database design is an ill-defined task. The final outcome i.e. the data model is defined in abstract terms, but there is no algorithm to find it. The tasks supported by other existing dialogue-based tutoring systems support are well-defined: (such as Mathematics, Physics) problem-solving is well-structured, and therefore the explanations that are expected from the learners can be clearly defined [1]. EER-Tutor allows the students to work on any part of the solution facilitating the ill-defined nature of the task. The constraint-based methodology (CBM) that is used to develop EER-Tutor does not impose any restrictions on which on the order at which a student arrives at a solution.

Our model for supporting dialogues consists of three parts: an error hierarchy, tutorial dialogues and rules for adapting them. The error hierarchy categorizes all error types in a domain. At the leaf level, an error type is associated with one or more violated constraints. Remediation is facilitated through dialogues, one of which is developed for each error type. In the case of multiple errors in a student solution, the hierarchy is traversed to select the error most suitable for discussion and the corresponding dialogue is then initiated. Finally, the adaptation rules are used to individualize the dialogues to suit the student's knowledge and reasoning skills by controlling their timing and the exact content. In response to the generated dialogue learners are able to provide answers by selecting an option from a list.

We evaluated the effectiveness of our model in an authentic classroom environment at the University of Canterbury in March 2010. The experimental group participants received adaptive dialogues that were customised based on their student models. The control group received nonadaptive dialogues regards of their knowledge level and the explanation skills. At the end of a single 2-hour session, the performance on pre- and post-tests indicate that the experimental group learned significantly more than their peers. The experimental group also learnt a significantly higher number of constraints.

In this interactive event, the participants will have the opportunity to solve problems in EERTutor and engage in dialogues. They will be able to experience how the dialogues are customised based on their knowledge level and their interactions with the dialogues.

\section{Reference}

[1] Weerasinghe, A., Mitrovic, A., Martin, B. Towards Individualized Dialogue Support for IllDefined Domains. IJAIED, Special Issue on Ill-Defined Domains, 2009. 19(4): p. 357-379. 